REVIEW

\title{
Apical Maxi-chloride channel from human placenta: 12 years after the first electrophysiological recordings
}

\author{
GLORIA RIQUELME
}

Programa de Fisiología y Biofísica, Instituto de Ciencias Biomédicas (ICBM), Facultad de Medicina, Universidad de Chile

\begin{abstract}
The Maxi-chloride channel was the first ion channel described by electrophysiological methods in placenta. Because it is difficult to access a complex epithelium such as the placenta for electrophysiological procedures, the studies of ion channels from placental membranes have been performed only very recently. It was only in 1993 that a direct demonstration of a high-conductance chloride channel in apical membranes of intact trophoblastic epithelium was mentioned, and two years later, the description of this channel was reported from purified placental apical membranes reconstituted into artificial lipid membranes suitable for patch-clamp recordings. This brief review comments on the work done with regard to the electrophysiological characterization and regulation of the large-conductance or "Maxi" chloride channel and its contribution to the development of a cellular model for syncytiotrophoblast ion transport.
\end{abstract}

Key words: apical membrane, chloride channel, human placenta.

\section{DEDICATION}

A Guayo, maestro sabio y hombre generoso de las ciencias y de la vida. Hacedor de ilusiones, enmendador de dificultades...

\section{INTRODUCTION}

Transport functions in the placenta have great importance for fetal growth and development. The human placental syncytiotrophoblast is a polarized epithelial structure, which forms the main barrier for materno-fetal exchange (Shennan et al., 1986). This epithelium is composed of large, multinucleated cells that result from the fusion of precursor cytotrophoblast cells, producing a syncytium. Thus, there is no paracellular route and all the solutes (water, nutrients such as amino acids, ions, etc.) must pass directly through both trophoblastic membranes. The analysis of transplacental transport implies the understanding of transport through both the fetal-facing basal membrane (BM) and the maternal-facing microvillous membrane (MVM). To develop a cellular model of syncytiotrophoblast ion transport, it has been necessary to characterize the ion channels present in both the apical and basal membranes. In placental syncytiotrophoblast, as in other epithelial cells, ion transport involving conductive pathways is associated with numerous functions, such as maintenance of membrane voltage, cell volume regulation, nutrient transport, and other placental processes (Birdsey et al., 1999; Birdsey et al., 1997; Clarson et al., 2001). There are several reports, using direct or indirect methods, which show the presence of cationic and anionic channels in these membranes, the most frequently reported among them is a large chloride channel.

\section{PLACENTAL TRANSPORT OF CHLORIDE IONS}

Chloride is the main anion of extracellular fluid in the fetus, as in the adult, but at all 
gestational ages, fetal chloride is 5 to 6 $\mathrm{mmol} / \mathrm{L}$ higher than in maternal blood. There are no differences in either sodium or potassium ion concentrations (Bissonnette et al., 1994).

The study of chloride transport mechanisms by the placenta has been performed mainly with isolated membrane vesicles prepared from term placentas. Chloride could enter and leave cells by a transport system coupled with $\mathrm{Na}^{+}, \mathrm{K}^{+}$, or both, in exchange for other anions or via conductive pathways (channels) (Stulc, 1997). Although there are a number of studies of chloride transport across the basal trophoblastic membrane, considerable interest has been focused on chloride transport in the apical membrane of the human placental syncytiotrophoblast microvillous vesicle membrane (MVM).

Historically, data from MVM vesicle flux studies have demonstrated that there are at least three different pathways for chloride flux across the MVM of the human placenta: one pathway via an exchanger and two via channels. A significant fraction of this flux, about $50 \%$, occurs by an electroneutral anion exchanger $\left(\mathrm{Cl}^{-} / \mathrm{HCO}_{3}\right)$ sensitive to DIDS (stilbene derivative 4, 4'diisothiocyanostilbene -2, 2'disulphonic acid) with similar properties to those of the monovalent anion exchanger in kidney, liver, and intestine (Dechecchi \& Cabrini, 1988; Davis et al., 1985). The remaining flux is dependent on membrane potential and several authors have suggested that it probably takes place through a conductive pathway (Shennan et al., 1986; Shennan, 1988; Boyd \& Shennan, 1986; Dechecchi \& Cabrini, 1988; Illsley et al., 1988). Subsequent detailed studies of chloride fluxes and ionic dependence of membrane potential have confirmed the presence of chloride conductive pathways in the microvillous membrane (Placchi et al., 1991; Illsley \& Sellers, 1992; Faller \& Ryan, 1992). These conductive pathways have been classified into two groups: a DPC (diphenylamine-2-carboxylic acid)sensitive $\mathrm{Cl}^{-}$conductance, and a DIDSsensitive $\mathrm{Cl}^{-}$conductance (Byrne et al., 1993; Boyd \& Vatish, 1994). It is difficult to tell from studies with vesicles which pathways are important in maternofetal exchange. However, it is interesting to note that DIDS, but not DPC, markedly reduces maternofetal clearance of chloride across the rat placenta perfused in situ (Stulc, 1997). During this period of flux studies, there also was considerable interest in the regulation of the chloride conductance in apical membrane vesicles of human placenta. It has been suggested that apical membrane chloride conductance is inhibited by protein kinase A-dependent phosphorylation (Placchi et al., 1991) and by unsaturated fatty acids such as arachidonic acid and linoleic acid (Faller \& Ryan, 1992; Boyd \& Vatish, 1994; Doolan \& Keenan, 1994). In order to differentiate the types of channels that are specifically regulated in placental membranes and to determine their biophysical properties, it was necessary to develop another line of work involving electrophysiological methods.

\section{PLACENTAL MAXI-CHLORIDE CHANNEL}

\section{The first steps: Single-channel recording}

The necessary next step would have to be single-channel recording. Nevertheless, this obvious jump from an indirect system, like the study of fluxes, to an experimental system that allowed the direct study of its functional characteristics was not an easy task.

Direct studies of ion transport mechanisms by human placenta or placental cotyledon have been limited because of the difficulties in gaining access to the epithelial membranes in whole tissue. In 1993, Brown et al. reported, for the first time, the use of the patch-clamp technique to provide direct access to the apical membrane of intact epithelia and to allow the investigation of its conductive ion transport (Brown et al., 1993). The authors studied ion channels using this method in the intact microvillous membrane of the term human placental syncytiotrophoblast. They described a chloride channel of highconductance, voltage-dependent open probability that was inhibited by DIDS, a 
predominant feature of the microvillous membrane. This work has been very important in the development of ways to study ion channels in human placental membranes. They assessed the possibility of applying electrophysiological methods in intact placental villous fragments and identified a chloride channel in the patches. However, the application of this technique directly is difficult because the placenta is a large, multinucleated cell, and the success rate in obtaining high resistance seals in situ is low. Brown et al. obtained high resistance seals in $30 \%$ of attempts in villi from over 50 placentae, and they recorded the channel in only a few of them.

Alternative methodologies are the reconstitution of ion channels from purified trophoblast plasma membranes, extracted from either normal placenta or pathological tissue, into artificial lipid membranes (Riquelme et al., 1995; Grosman et al., 1997), or their functional transplantation into Xenopus laevis oocytes (Ivorra et al., 2002). Both methods require highly purified membranes. At the moment, the development of improved methods for the isolation and purification of these membranes provides good material for the investigation of the structure and function of placental ion channels (Glazier et al., 1988; Glazier et al., 1990; Illsley et al., 1990; Jimenez et al., 2004).

In 1995, Riquelme et al. reported the first description of an ion channel recording from human term placental trophoblast reconstituted into giant liposomes, a preparation suitable for patch-clamp recording of single-channel ion currents. Human placental apical membrane was purified by differential and gradient centrifugation and fused into small liposomes. Giant liposomes were then generated by a method of cycle dehydration and rehydration of lipid vesicles (Riquelme et al., 1990; Riquelme et al., 1995). The most frequently observed activity in stable patches was a large-conductance chloride channel with biophysical characteristics similar to those described by Brown et al. in apical membranes of the intact fragments of villous tissue (Brown et al., 1993). Grosman et al. in 1997 reported the reconstitution of ionic channels on planar lipid bilayers from apical membrane and one of them was also a chloride channel with high conductance (Grosman et al., 1997). This was followed by numerous works that reported the characteristics, regulation, and possible function of this type of placental channels.

\section{Biophysical characteristics}

The features of the single-channel recordings are flickering to transitional current levels between the fully open and fully closed states, showing that the open channel opens to a number of intermediate subconductance states. The channel has a large conductance; the mean slope conductance ranged from 200 to $350 \mathrm{pS}$ when only the dominant current level was used, ignoring any subconductance levels (Brown et al., 1993; Riquelme et al., 1995; Grosman et al., 1997). The channel is selective for anions over cations, including amino acids such as glutamate and aspartate, and the permeability ratio for $\mathrm{Cl}^{-}$over $\mathrm{Na}^{+}$was 7 (Riquelme et al., 1995). Another characteristic feature of the channel is the voltage dependence of its opening. The open probability versus voltage relationship could be described by a bell-shaped curve. The placental chloride channel is predominantly open at potentials between $\pm 30 \mathrm{mV}$ in inside-out patches from intact placental fragments (Brown et al., 1993) and at \pm 50 $\mathrm{mV}$ in excised patch from giant liposomes containing placental apical membranes (Riquelme et al., 1995; Riquelme \& Parra, 1999). More extreme potentials, in either a positive or negative direction, induce channel closure. This channel is inhibited by DIDS (Riquelme \& Parra, 1999; Brown et al., 1993), but is insensitive to DPC, another chloride channel blocker. These properties are similar to those described for a family of chloride channels with conductances ranging between 200 and 500pS classified as Maxichloride channels. Maxi-chloride channels have been identified in secreting and absorbing epithelia (Halm \& Frizzell, 1992) and also in some non-epithelial cell types (Blatz \& Magleby, 1983; Gray et al., 1984; Nelson et al., 1984; Nobile \& Galietta, 
1988). In summary, normal term placenta has a Maxi-chloride channel in the apical membrane of the syncytiotrophoblast comparable to other Maxi-chloride channels described in epithelial and non-epithelial cells.

According to early studies using microvillous membrane vesicles (Shennan et al., 1986; Dechecchi \& Cabrini, 1988; Illsley et al., 1988), the presence of chloride channels in the apical membrane could be underlying two conductive pathways for chloride, one of them sensitive to DIDS and the other one sensitive to DPC. The Maxichloride channel in placenta is blocked by DIDS but is unaffected by DPC, therefore the Maxi-chloride channel is a possible molecular candidate responsible for the DIDS-sensitive anion conductance in the apical syncytiotrophoblast plasma membrane detected in the chloride influx studies.

REGULATION OF PLACENTAL MAXI-CHLORIDE CHANNEL

\section{Regulation by fatty acids}

Unsaturated fatty acids have been shown to inhibit placental chloride channels in accordance with the inhibition observed in chloride channels in cultured airway epithelia (Anderson \& Welsh, 1990; Hwang et al., 1990) and large-conductance chloride channels in L6 myoblasts (Zachar \& Hurnak, 1994). In placenta, the interest in studying the regulation of chloride conductances by fatty acids in apical membrane vesicles of syncytiotrophoblast has been present in previous studies using flux methods. Assays of radioactive chloride flux have demonstrated that fatty acids, such as arachidonic acid and linoleic acid, inhibit DIDS-sensitive chloride conductive pathways in microvillous membranes of human placenta (Boyd \& Vatish, 1994; Doolan \& Keenan, 1994; Faller \& Ryan, 1992). Late, it was demonstrated that the Maxi-chloride channel reconstituted in giant liposomes is sensitive to fatty acids. Low micromolar concentrations of arachidonic acid inhibit
Maxi-chloride channels in a reversible and dose-dependent manner. Arachidonic acid decreased both the single-channel conductance and open probability. Other cis unsaturated fatty acids, such as oleic and linoleic acids, showed a similar blockade (Riquelme \& Parra, 1999). In addition to these results, this experimental approach (a reconstituted system devoid of enzymes and metabolites), has demonstrated that the fatty acids act directly on the channel rather than acting through the mediation of a metabolic process.

\section{Regulation by steroid hormones}

Steroid hormones modulate the placental Maxi-chloride channel, a fact that has been observed in other chloride channels, such as volume-regulated chloride channels in epithelial cells (Zhang et al., 1994), a Maxichloride channel in fibroblasts, neuroblastoma, and porcine aortic endothelial cells (Hardy \& Valverde, 1994; Diaz et al., 2001; Li et al., 2000). The Maxi-chloride channel, in excised patches from giant liposomes containing placental apical membrane, is inhibited in a potentialdependent manner by $17 \beta$-Estradiol. This effect is seen at micromolar concentrations, similar to the concentrations of this hormone detected in blood of pregnant women. The addition of $1 \mu \mathrm{M} 17 \beta$ Estradiol to the bath solution shifted the bell shaped curve that describes the open probability voltage relationship to a more positive potential, the open probability decreased at negative potentials, however the single-channel conductance was unaffected. The stereoisomer $17 \alpha$-Estradiol did not affect the chloride current. Tamoxifen, an antiestrogen, also showed inhibition, but in a voltage-independent manner (Henriquez \& Riquelme, 2003). These results suggest a direct interaction of estrogen with the channel, similar to the direct interaction of $17 \beta$-Estradiol with a Maxi-K ${ }^{+}$channel (Valverde et al., 1999)

\section{Regulation by annexin 6}

Annexins are a family of calciumdependent, phospholipids-binding proteins, 
and several reports show that specific members of this family participate in the regulation of ionic channels in different cells. More specifically, annexin 6 regulates the Maxi-chloride channel from apical membranes. Riquelme et al. (2004) showed that annexin 6 is present in the purified apical membranes from human syncytiotrophoblast, and it binds to the apical placental membranes in two ways: in a calcium-dependent phospholipids-binding manner, as expected, and also in a calciumindependent fashion. The neutralization of annexin 6 directly by anti-annexin 6 antibodies in excised patches from giant liposomes containing apical membranes showed that the biophysical properties of the Maxi-chloride channel are affected by this protein. The single-channel slope conductance decreased by $50 \%$ and the open probability also was affected. The open probability did not decrease at higher voltage steps, neither in the positive nor the negative direction, while normally voltage induces channel closure. The authors suggest that endogenous annexin 6 could regulate the Maxi-chloride channel (Riquelme et al., 2004). Considering that in other cells, specifically in smooth muscle cells, annexin 6 participates in the formation of reversible cytoskeleton complexes (Babiychuk et al., 1999), these results seem consistent with those results reported for other Maxi-chloride channels, suggesting that the cytoskeleton participates in the regulation of the Maxi-chloride channels. This regulation could explain why the activity of this family of channels is detected mainly in isolated patches or in general when there is disruption of the cytoskeleton (Strange et al., 1996).

MAXI-CHLORIDE CHANNEL FROM PRE-ECLAMPTIC HUMAN PLACENTA

There is only one report referring to the behavior of this channel from pathological placenta. Bernucci et al. showed the first evidence of a functionally altered ionic channel from placental syncytiotrophoblast from preeclamptic pregnancies (Bernucci et al., 2003). Pre-eclampsia, a frequent pathology of pregnancy exerts a great impact on fetal morbidity and mortality. When reconstituted into giant liposomes suitable for electrophysiological studies, the characteristics of the placental chloride channel from pre-eclamptic pregnancies were different from those observed in normal pregnancies. The channels were selective for anions over cations, had multiple subconductance states, and were inhibited by DIDS. They also had a large conductance but, unlike the normal chloride channel, the predominant conductance was lower than $200 \mathrm{pS}$. Also, the voltage dependence of the open probability changed; the relationship between both exhibited a flattened curve instead of the bell-shaped curve of normal placenta.

PLACENTAL MAXI-CHLORIDE CHANNEL: UNANSWERED QUESTIONS

\section{A channel looking for its function}

There is growing interest in identifying the chloride conductive pathways in trophoblastic membranes because in placental syncytiotrophoblast, like in other epithelial cells, ion transport involving conductive pathways is associated with numerous epithelial functions, such as maintenance of membrane voltage, cell volume regulation, solute transport, etc. However, the specific ion channels involved in these and other placental processes are yet unknown. The inexplicable fact it is that the role of the Maxi-chloride channel characterized in the apical membrane is still unclear.

It has been reported in placenta, using indirect methods, that a $\mathrm{Ba}^{2+}$-sensitive $\mathrm{K}^{+}$ conductance and a DIDS-sensitive anion conductance contribute to the resting potential of the syncytiotrophoblast microvillous membrane and that these conductances also are involved in volume regulation (Birdsey et al., 1999; Clarson et al., 2001). These studies have been a significant step towards characterizing the participation of ion conductances in these important functions using intact placental villi. A Maxi-chloride channel is a possible 
molecular candidate for the DIDS-sensitive anion conductance mentioned above in apical syncytiotrophoblast plasma membranes. Clearly, the understanding of the interaction between solute transport and changes in electrical driving forces is necessary for the development of a model for the mechanisms of materno-fetal exchange. Materno-fetal solute transport across the syncytiotrophoblast implies nutrient transport which is driven, at least partially, by the electrochemical gradient, a determinant of which is the resting membrane potential (Sibley et al., 1998). Among the nutrients in placenta, taurine is the most abundant amino acid; its concentration is 100 to 200 -fold that of maternal blood. For the fetus, taurine is an essential amino acid and appears to be vital for fetal growth and for CNS (Central Nervous System) development. It is derived from the maternal circulation via the placenta, indicating the need for a highly efficient transplacental transport via transporters as is already known and possibly also via conductive pathways. Interestingly there are several studies in fragments of villous tissue from human placenta, that have shown volume-activated amino acid efflux via a pathway sensitive to classic inhibitors of anionic transporters (DIDS, NPPB, and niflumic acid). This is consistent with the presence of the volumeactivated $\mathrm{Cl}^{-}$channels in placental tissue (Shennan \& McNeillie, 1995a; Shennan \& McNeillie, 1995b). In particular, the transport of taurine in response to a hyposmotic challenge in placental tissue explants has been examined. Some authors propose that volume-activated taurine efflux from placental tissue is mediated by a chloride channel (Shennan et al., 1993; Shennan, 1999). There is evidence that the Maxi-chloride channel is activated by cell swelling in neuroblastoma cells, rat cortical astrocytes, and a renal cortical collecting duct cell line, among others (Strange et al., 1996; Kirk \& Strange, 1998). Thus, the apical Maxi-chloride channel from placenta also is a possible candidate for the chloride and amino acid efflux in response to hyposmotic conditions, including the taurine pathways.

\section{A channel with an unknown structure}

There are several questions that involve the knowledge of the molecular structure of the channel. For example: the open question regarding whether the channels from apical membrane from pre-eclamptic placenta are molecular variants of the Maxi-chloride channels from normal placentas or whether the normal channel is functionally modified in this pathology. Another example is the case of the regulation by annexin 6: the complementary experiments could be the biochemical demonstration of an association of Maxi-chloride channels with annexin 6, determining whether annexin 6 copurifies with the Maxi-chloride channel. But these questions are not possible to answer now because the molecular identity of Maxi-chloride channels is not clear yet. Large conductance chloride (Maxi- $\mathrm{Cl}^{-}$) currents have been recorded in several types of cells, but there is still little information on the molecular nature of the channel underlying this conductance, while the search for its molecular identity has continued, with irregular success, over all these years. The fact that the electrophysiological properties of the Maxichloride channel resemble those of the mitochondrial voltage-dependent anion channel (VDAC) (Colombini, 1980) encouraged some investigators to assume that the two channels were one and the same protein. This hypothesis was based on early observations suggesting the presence of the VDAC protein on the plasma membrane (Thinnes et al., 1989; Dermietzel et al., 1994; Buettner et al., 2000; Bahamonde et al., 2003), but was questioned by others (Yu et al., 1995; Yu \& Forte, 1996). Recently Suzuki and Mizuno have reported that a gene located in Drosophila flightless has a structure similar to those of known channels and that human homologues of tweety (hTTYH1-3) are novel Maxi- chloride channels. In this study, the authors showed that the hTTYH family possesses five or six transmembrane segments encoding a large conductance chloride channel (Suzuki \& Mizuno, 2004).

In summary, regarding the structure of the channel, there is no answer yet to this 
question for the Maxi-chloride channel in general nor for the placental Maxi-chloride channel.

\section{CONCLUSIONS}

The development of a direct experimental approach to study the ionic conductances present in syncytiotrophoblast has improved the knowledge of the biophysical properties and regulation of the ion channels in the membranes of this tissue. This could be a contribution to advancing the understanding of the function of both normal and pathological placenta.

In particular, all the information reported on the placental Maxi-chloride channel suggests that this channel plays a role in a cellular model of syncytiotrophoblast ion transport, although its function is not yet clear.

Functionally, the control of these channels by fatty acids may be important in regulating epithelial electrolyte transport and nutrient delivery to the fetus, as suggested by the importance of arachidonic acid metabolism in placental tissue. Abnormal regulation of cell fatty acid production may be the cause of some of the pathological conditions observed during the gestational period.

In placenta, there is evidence that steroid hormones, which are present in high concentrations during pregnancy in humans, have some influence in secretory and absorbing processes, but the molecular mechanisms remain elusive. From a functional point of view, the control of Maxi-chloride channels by steroid hormones may be of great importance in placental physiology, because this effect could help clarify the mechanism involved in the regulation of important functions, such as chloride secretion. It is possible to make similar projections with the regulation of the Maxi-chloride channel by annexin 6, and this may open new fields of study regarding the role of this interaction in complications of pregnancy, such as preeclampsia, in which the Maxi-chloride channel has been shown to have a decreased conductance and a flattened open-probability curve similar to that observed in experiments in normal placentas in which annexin 6 has been neutralized. In recent years, there has been an important increase in reports of ionic channel activity dysfunctions associated to diverse pathologies (Ashcroft, 2000). Defects in ion channel function are presently of great interest, not only because of their capability of generating therapeutical strategies, but also because such alterations aid in the understanding of ion channel function.

In conclusion, over the last 12 years there have been a number of reports that have demonstrated the presence of apical Maxi-chloride channel in human placenta and its regulation by fatty acids, steroid hormones, and annexin 6 . This channel also has been described in placentas from preeclamptic pregnancies with some differences in its biophysical characteristics with respect to channels from normal placenta. All this data, without doubt, could be important in the generation of a model of solute transfer across the placenta. Although we are still very far from having all the answers about placental ionic transport by conductive pathways, the available information and the experimental tools to reach this objective have improved remarkably.

\section{ACKNOWLEDGEMENTS}

The original work by the author has been supported by grants FONDECYT (\#1040546 - \# 1000647 and \#1970235). I also thank E. Mancilla M.D. for critically reading the manuscript.

\section{REFERENCES}

ANDERSON MP \& WELSH MJ (1990) Fatty acids inhibit apical membrane chloride channels in airway epithelia. Proc Natl Acad Sci USA 87(18): 7334-733

ASHCROFT F (2000) Ion channels and disease. San Diego, California, USA: Academic press

BABIYCHUK EB, PALSTRA RJ, SCHALLER J, KAMPFER U, DRAEGER A (1999) Annexin VI participates in the formation of a reversible, membrane-cytoskeleton complex in smooth muscle cells. J Biol Chem 274(49): 35191-35195 
BAHAMONDE MI, FERNÁNDEZ-FERNÁNDEZ JM, GUIX FX, VÁZQUEZ E, VALVERDE MA (2003) Plasma membrane voltage-dependent anion channel mediates antiestrogen-activated maxi $\mathrm{Cl}$ - currents in $\mathrm{C} 1300$ neuroblastoma cells. J Biol Chem 278(35): 33284-33289

BERNUCCI L, UMANA F, LLANOS P, RIQUELME G (2003) Large chloride channel from pre-eclamptic human placenta. Placenta 24(8-9): 895-903

BIRDSEY TJ, BOYD RD, SIBLEY CP, GREENWOOD SL (1997) Microvillous membrane potential (Em) in villi from first trimester human placenta: Comparison to Em at term. Am J Physiol 273(4 Pt 2): R1519-R1528

BIRDSEY TJ, BOYD RD, SIBLEY CP, GREENWOOD SL (1999) Effect of hyposmotic challenge on microvillous membrane potential in isolated human placental villi. Am J Physiol 276(5 Pt 2): R1479-R1488

BISSONNETTE JM, WEINER CP, POWER GG, JR (1994) Amino acid uptake and chloride conductances in human placenta. Placenta 15(4): 445-446

BLATZ AL, MAGLEBY KL (1983) Single voltagedependent chloride-selective channels of large conductance in cultured rat muscle. Biophys J 43(2): 237-241

BOYD CA SHENNAN DB (1986) Human placental sulphate transport: Studies on chorionic trophoblast brush border membrane vesicles. J Physiol 377: 15-24

BOYD CA \& VATISH M (1994) Regulation of conductive chloride transport in human placental brush border membrane vesicles. Jpn J Physiol 44 Suppl 2: S273S280

BROWN PD, GREENWOOD SL, ROBINSON J, BOYD RD (1993) Chloride channels of high conductance in the microvillous membrane of term human placenta. Placenta 14(1): 103-115

BUETTNER R, PAPOUTSOGLOU G, SCEMES E, SPRAY DC, DERMIETZEL R (2000) Evidence for secretory pathway localization of a voltage-dependent anion channel isoform. Proc Natl Acad Sci USA 97(7): 3201-3206

BYRNE S, GLAZIER JD, GREENWOOD SL, MAHENDRAN D, SIBLEY CP (1993) Chloride transport by human placental microvillous membrane vesicles. Biochim Biophys Acta 1153(1): 122-126

CLARSON LH, GREENWOOD SL, MYLONA P, SIBLEY CP (2001) Inwardly rectifying $K(+)$ current and differentiation of human placental cytotrophoblast cells in culture. Placenta 22(4): 328-336

COLOMBINI M (1980) Structure and mode of action of a voltage dependent anion-selective channel (VDAC) located in the outer mitochondrial membrane. Ann NY Acad Sci 341: 552-563

DAVIS B, SHENNAN DB, BOYD CA (1985) Chloride transport in cystic fibrosis placenta. Lancet 1(8425): 392-393

DECHECCHI MC, CABRINI G (1988) Chloride conductance in membrane vesicles from human placenta using a fluorescent probe. Implications for cystic fibrosis. Biochim Biophys Acta 945(2): 113-120

DERMIETZEL R, HWANG TK, BUETTNER R, HOFER A, DOTZLER E, KREMER M, DEUTZMANN R, THINNES FP, FISHMAN GI, SPRAY DC, SIEMEN D (1994) Cloning and in situ localization of a brainderived porin that constitutes a large-conductance anion channel in astrocytic plasma membranes. Proc Natl Acad Sci USA 91(2): 499-503

DÍAZ M, BAHAMONDE MI, LOCK H, MUNOZ FJ, HARDY SP, POSAS F, VALVERDE MA (2001) Okadaic acid-sensitive activation of Maxi Cl(-) channels by triphenylethylene antioestrogens in C1300 mouse neuroblastoma cells. J Physiol 536(Pt 1): 79-88
DOOLAN CM, KEENAN AK (1994) Inhibition by fatty acids of cyclic AMP-dependent protein kinase activity in brush border membranes isolated from human placental vesicles. Br J Pharmacol 111(2): 509-514

FALLER D, RYAN MP (1992) Factors affecting chloride conductance in apical membrane vesicles from human placenta. J Membr Biol 130(3): 227-239

GLAZIER JD, JONES CJ, SIBLEY CP (1988) Purification and $\mathrm{Na}$ - uptake by human placental microvillus membrane vesicles prepared by three different methods. Biochim Biophys Acta 945(2): 127-134

GLAZIER JD, JONES CJ, SIBLEY CP(1990) Preparation of plasma membrane vesicles from the rat placenta at term and measurement of Na+ uptake. Placenta 11(5): 451-463

GRAY PT, BEVAN S, RITCHIE JM (1984) High conductance anion-selective channels in rat cultured Schwann cells. Proc R Soc Lond B Biol Sci 221(1225): 395-409

GROSMAN C, MARIANO MI, BOZZINI JP, REISIN IL (1997) Properties of two multisubstate Cl- channels from human syncytiotrophoblast reconstituted on planar lipid bilayers. J Membr Biol 157(1): 83-95

HALM DR, FRIZZELL RA (1992) Anion permeation in an apical membrane chloride channel of a secretory epithelial cell. J Gen Physiol 99(3): 339-366

HARDY SP, VALVERDE MA (1994) Novel plasma membrane action of estrogen and antiestrogens revealed by their regulation of a large conductance chloride channel. FASEB J 8(10): 760-765

HENRÍQUEZ M, RIQUELME G (2003) 17beta-estradiol and tamoxifen regulate a maxi-chloride channel from human placenta. J Membr Biol 191(1): 59-68

HWANG TC, GUGGINO SE, GUGGINO WB (1990) Direct modulation of secretory chloride channels by arachidonic and other cis unsaturated fatty acids. Proc Natl Acad Sci USA 87(15): 5706-5709

ILLSLEY NP, GLAUBENSKLEE C, DAVIS B VERKMAN AS (1988) Chloride transport across placental microvillous membranes measured by fluorescence. Am J Physiol 255(6 Pt 1): C789-C797

ILLSLEY NP, SELLER MC (1992) Ion conductances in the microvillous and basal membrane vesicles isolated from human placental syncytiotrophoblast. Placenta 13(1): 25-34

ILLSLEY NP, WANG ZQ, GRAY A, SELLERS MC, JACOBS MM (1990) Simultaneous preparation of paired, syncytial, microvillous and basal membranes from human placenta. Biochim Biophys Acta 1029(2): 218-226

IVORRA I, HENRÍQUEZ M, LAX P, RIQUELME G, MORALES A (2002) Functional transplantation of chloride channels from the human syncytiotrophoblast microvillous membrane to Xenopus oocytes. Pflugers Arch 444(6): 685-691

JIMÉNEZ V, HENRÍQUEZ M, LLANOS P, RIQUELME G (2004) Isolation and purification of human placental plasma membranes from normal and pre-eclamptic pregnancies. A comparative study. Placenta 25(5): 422437

KIRK K, STRANGE K (1998) Functional properties and physiological roles of organic solute channels. Annu Rev Physiol 60: 719-739

LI Z, NIWA Y, SAKAMOTO S, CHEN X, NAKAYA Y (2000) Estrogen modulates a large conductance chloride channel in cultured porcine aortic endothelial cells. J Cardiovasc Pharmacol 35(3): 506-510

NELSON DJ, TANG JM, PALMER LG (1984) Singlechannel recordings of apical membrane chloride conductance in A6 epithelial cells. J Membr Biol 80(1): 81-89 
NOBILE M, GALIETTA LJ (1988) A large conductance $\mathrm{Cl}$ - channel revealed by patch-recordings in human fibroblasts. Biochem Biophys Res Commun 154(2): 719-726

PLACCHI P, LOMBARDO R, TAMANINI A, BRUSA P BERTON G, CABRINI G (1991) cAMP-dependent protein kinase inhibits the chloride conductance in apical membrane vesicles of human placenta. J Membr Biol 119(1): 25-32

RIQUELME G, LLANOS P, TISCHNER E, NEIL J, CAMPOS B (2004) Annexin 6 modulates the maxichloride channel of the apical membrane of syncytiotrophoblast isolated from human placenta. J Biol Chem 279(48): 50601-50608

RIQUELME G, LÓPEZ E, GARCÍA-SEGURA LM, FERRAGUT JA, GONZÁLEZ-ROS JM (1990) Giant liposomes: A model system in which to obtain patchclamp recordings of ionic channels. Biochemistry 29(51): 11215-11222

RIQUELME G, PARRA M (1999) Regulation of human placental chloride channel by arachidonic acid and other cis unsaturated fatty acids. Am J Obstet Gynecol 180(2 Pt 1): 469-475

RIQUELME G, STUTZIN A, BARROS LF, LIBERONA JL (1995) A chloride channel from human placenta reconstituted into giant liposomes. Am J Obstet Gynecol 173(3 Pt 1): 733-738

SHENNAN DB (1988) Selenium (selenate) transport by human placental brush border membrane vesicles. Br J Nutr 59(1): 13-19

SHENNAN DB (1999) Properties of volume-activated taurine and iodide efflux from term human placental tissue. Placenta 20(5-6): 485-491

SHENNAN DB, DAVIS B, BOYD CA (1986) Chloride transport in human placental microvillus membrane vesicles. I. Evidence for anion exchange. Pflugers Arch 406(1): 60-64

SHENNAN DB, MCNEILLIE SA (1995a) Volumeactivated amino acid efflux from term human placental tissue: Stimulation of efflux via a pathway sensitive to anion transport inhibitors. Placenta 16(3): 297-308

SHENNAN DB, MCNEILLIE SA (1995b) Volumeactivated solute transport in human placental tissue. Biochem Soc Trans 23(1): 25S
SHENNAN DB, MCNEILLIE SA, CURRAN DE (1993) Stimulation of taurine efflux from human placental tissue by a hypoosmotic challenge. Exp Physiol 78(6): 843-846

SIBLEY CP, BIRDSEY TJ, BROWNBILL P, CLARSON LH, DOUGHTY I, GLAZIER JD, GREENWOOD SL, HUGHES J, JANSSON T, MYLONA P, NELSON DM, POWELL T (1998) Mechanisms of maternofetal exchange across the human placenta. Biochem Soc Trans 26(2): 86-91

STRANGE K, EMMA F, JACKSON PS (1996) Cellular and molecular physiology of volume-sensitive anion channels. Am J Physiol 270(3 Pt 1): C711-C730

STULC J (1997) Placental transfer of inorganic ions and water. Physiol Rev 77(3): 805-836

SUZUKI M, MIZUNO A (2004) A novel human $\mathrm{Cl}(-)$ channel family related to Drosophila flightless locus. J Biol Chem 279(21): 22461-22468

THINNES FP, GOTZ H, KAYSER H, BENZ R, SCHMIDT WE, KRATZIN HD, HILSCHMANN N (1989) [Identification of human porins. I. Purification of a porin from human B-lymphocytes (Porin 31HL) and the topochemical proof of its expression on the plasmalemma of the progenitor cell.] Biol Chem Hoppe Seyler 370(12): 1253-1264

VALVERDE MA, ROJAS P, AMIGO J, COSMELLI D, ORIO P, BAHAMONDE MI, MANN GE, VERGARA C, \& LATORRE R (1999) Acute activation of Maxi-K channels (hSlo) by estradiol binding to the beta subunit. Science 285(5435): 1929-1931

YU WH \& FORTE M (1996) Is there VDAC in cell compartments other than the mitochondria? J Bioenerg Biomembr 28(2): 93-100

YU WH, WOLFGANG W, FORTE M (1995) Subcellular localization of human voltage-dependent anion channel isoforms. J Biol Chem 270(23): 13998-14006

ZACHAR J, HURNAK O (1994) Arachidonic acid blocks large-conductance chloride channels in L6 myoblasts. Gen Physiol Biophys 13(3): 193-213

ZHANG JJ, JACOB TJ, VALVERDE MA, HARDY SP, MINTENIG GM, SEPÚLVEDA FV, GILL DR, HYDE SC, TREZISE AE, HIGGINS CF (1994) Tamoxifen blocks chloride channels. A possible mechanism for cataract formation. J Clin Invest 94(4): 1690-1697 
